\title{
Atmospheric muons: experimental aspects
}

\author{
S. Cecchini ${ }^{1}$ and M. Spurio ${ }^{1,2}$ \\ ${ }^{1}$ INFN, sezione di Bologna, Viale Berti Pichat 6/2, 40100 Bologna, Italy \\ ${ }^{2}$ Dipartimento di Fisica dell'Università di Bologna, Viale Berti Pichat 6/2, 40100 Bologna, Italy
}

Correspondence to: M. Spurio (maurizio.spurio@unibo.it)

Received: 17 July 2012 - Published in Geosci. Instrum. Method. Data Syst. Discuss.: 20 August 2012

Revised: 17 October 2012 - Accepted: 2 November 2012 - Published: 21 November 2012

\begin{abstract}
We present a review of atmospheric muon flux and energy spectrum measurements over almost six decades of muon momentum. Sea level and underground/water/ice experiments are considered. Possible sources of systematic errors in the measurements are examined. The characteristics of underground/water muons (muons in bundle, lateral distribution, energy spectrum) are discussed. The connection between the atmospheric muon and neutrino measurements are also reported.
\end{abstract}

\section{Introduction}

Primary cosmic rays (CRs) are particles accelerated at astrophysical sources continuously bombarding the Earth. When entering the Earth's atmosphere, primary CRs interact with the air nuclei and produce fluxes of secondary, tertiary (and so on) particles. All these particles together create a cascade, called air shower. As the cascade develops longitudinally the particles are less and less energetic since the energy of the incoming CR is split and redistributed among more and more participants. The transverse momenta acquired by the secondaries cause the particles to spread laterally as they propagate in the target. Muons predominantly originate from the decay of secondary charged pions and kaons. The most important decay channels and respective branching ratios are

$$
\begin{aligned}
& \pi^{ \pm} \rightarrow \mu^{ \pm}{ }_{v_{\mu}}^{(-)}(\sim 100 \%) \\
& K^{ \pm} \rightarrow \mu^{ \pm}{\stackrel{(-)}{v_{\mu}}}^{(\sim 63.5 \%) .}
\end{aligned}
$$

Atmospheric muons are the most abundant charged particles arriving at sea level and the only ones able to penetrate deeply underground. The reason relies on their small energy loss in the whole atmosphere $(\sim 2 \mathrm{GeV})$, the relatively long lifetime and the fairly small interaction cross section. Because of the close relation between muon and neutrino production, the parameters characterizing muon physics can provide important information on atmospheric neutrino flux.

An important parameter to describe the interactions and the subsequent propagation of the particles produced is the atmospheric depth $X$, measured in $\mathrm{g} \mathrm{cm}^{-2}$, defined as the integral in altitude of the atmospheric density above the observation level $h$ :

$X=\int_{h}^{\infty} \rho\left(h^{\prime}\right) d h^{\prime} \simeq X_{0} e^{-h / h_{0}}$.

In the last step, an approximation for an isothermal atmosphere was used, where $X_{0}=1030 \mathrm{~g} \mathrm{~cm}^{-2}$ is the atmospheric depth at sea level and $h_{0} \simeq 8.4 \mathrm{~km}$ is the scale height (for mid latitudes) in the atmosphere. Equation (2) is valid for vertically incident particles. For zenith angles $<60^{\circ}$, for which the Earth surface can be approximated as flat, the atmospheric depth is scaled with $1 / \cos \theta$, giving the slant depth. For larger zenith angles, the curvature of the Earth has to be accounted for. The atmospheric profile gives a total horizontal atmospheric depth of about $36000 \mathrm{~g} \mathrm{~cm}^{-2}$.

The air shower is described by a set of coupled cascade equations with boundary conditions at the top of the atmosphere to match the primary spectrum. Using the transport equations, analytic expressions of the cascade can be constructed. The solutions of these equations allow to compute the differential particle flux anywhere within the atmospheric target. Some approximate analytic solutions are valid in the limit of high energies (Gaisser, 1990, 2002; Lipari, 1993). Numerical or Monte Carlo calculations are needed to account accurately for decay and energy loss processes, and for the 
energy-dependences of the cross sections and of the primary spectral index. The nucleon mean free path $\lambda_{\mathrm{N}}$ in the atmosphere is given (in $\mathrm{g} \mathrm{cm}^{-2}$ units) by

$\lambda_{\mathrm{N}}=\frac{A m_{\mathrm{p}}}{\sigma_{\mathrm{N}}^{\text {air }}}$

where $\sigma_{\mathrm{N}}^{\text {air }}$ is the interaction cross section of nucleon in air, $A$ is the mean mass number of air nuclei and $m_{\mathrm{p}}$ the proton mass. For nucleons in the TeV range, $\sigma_{\mathrm{N}}^{\text {air }} \simeq 300 \mathrm{mb}$. In the context of air shower development, the energy-dependent cross section for an inelastic collision of a nucleon with an air nucleus is assumed to be constant. The atmosphere of the Earth consists mainly of nitrogen and oxygen: the interaction target for the primary beam is half protons and half neutrons. Assuming an average atmospheric nucleus with $A \sim 14.5, \lambda_{\mathrm{N}} \simeq 80 \mathrm{~g} \mathrm{~cm}^{-2}$. The total vertical atmospheric depth is about $1000 \mathrm{~g} \mathrm{~cm}^{-2}$ and it corresponds to more than 11 interaction lengths.

Most muons are produced through processes (Eqs. 1a and $1 \mathrm{~b}$ ) high in the atmosphere in the first few interaction lengths. The decay mean free path of pions $d_{\pi}$ in units of slant depth, is defined as

$$
\frac{1}{d_{\pi}}=\frac{m_{\pi} c^{2} h_{0}}{E c \tau_{\pi} X \cos \theta}=\frac{\epsilon_{\pi}}{E X \cos \theta}
$$

where $E, m_{\pi}, \tau_{\pi}$ are the pion energy, mass and lifetime, respectively. A similar relation holds for the kaon. Decay or interaction dominates depending on whether $1 / d_{\pi}$ or $1 / \lambda_{\pi}$ is larger. $\lambda_{\pi}$ is defined through Eq. (3) replacing $\sigma_{\mathrm{N}}$ with $\sigma_{\pi}$. At the critical energy $E=\epsilon_{\pi}=m_{\pi} c^{2} h_{0} / \tau_{\pi} c=115 \mathrm{GeV}$ the interaction probability in the atmosphere equals the decay probability.

As for the pion, all long-lived unstable particles (Braibant et al., 2012) are subject to competition between interaction and decay as they propagate in the atmosphere. The probability for either process to occur depends on the lifetime of the particle and is a function of its kinetic energy and on the local atmospheric density, which is a function of altitude. This interrelationship is responsible for the zenith angle enhancement of the bulk of the muons in air showers.

The zenith angle enhancement phenomenon does not affect the distribution of muons produced in semileptonic decays of charmed mesons, like $D^{ \pm}, D^{0}$ and others. As the lifetime of charmed particles is smaller than $\sim 10^{-12} \mathrm{~s} \mathrm{(prompt}$ decays), they yield so-called prompt (or direct) muons that are in general highly energetic for kinematic reasons. Since the production cross section of charmed mesons in protonnucleon interactions is rather small, $D$ decays contribute significantly only at very high energies.

\section{Cosmic rays at the sea level}

Muons are the dominant component of charged particles at sea level. The integral fluxes of particles arriving at

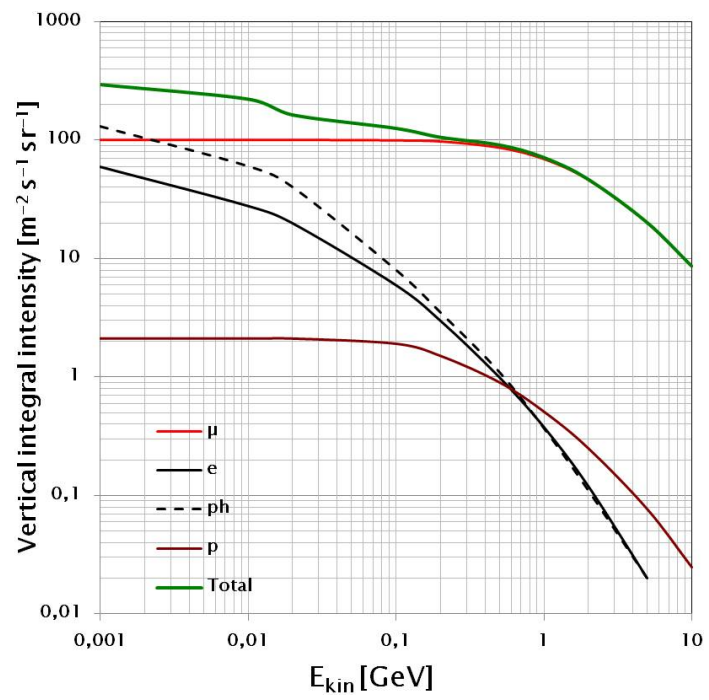

Fig. 1. Integral fluxes averaged over the 11-yr solar cycle of $\mu, e, p$ and photons (ph) arriving at geomagnetic latitudes $\sim 40^{\circ}$ vs. their kinetic energy.

geomagnetic latitudes $\sim 40^{\circ}$ vs. their kinetic energy are presented in Fig. 1. Fluxes are averaged over the 11-yr solar cycles. The muon flux with $E_{\mu}>1 \mathrm{GeV}$ through a horizontal area amounts to roughly one particle per $\mathrm{cm}^{2}$ and per minute: $I_{\mathrm{v}}\left(E_{\mu}>1 \mathrm{GeV}\right) \sim 70 \mathrm{~m}^{-2} \mathrm{~s}^{-1} \mathrm{sr}^{-1}$ (Grieder, 2010).

The muon energy spectrum at sea level is a direct consequence of the meson source spectrum. Below the few $\mathrm{GeV}$ energy range, the muon decay probability cannot be neglected. A muon of $1 \mathrm{GeV}$ has a Lorentz factor $\gamma=E_{\mu} / m_{\mu} c^{2} \sim 10$ and it has a mean decay length $d_{\mu}=\gamma \tau_{\mu} c \sim 6 \mathrm{~km}$. Since pions are typically produced at altitudes of $15 \mathrm{~km}$ and decay relatively fast (for $\gamma=10$ the decay length is $d_{\pi} \sim 78 \mathrm{~m}$, which is almost the same value of $\lambda_{\pi}$ ), the daughter muons do not reach the sea level but rather decay themselves or get absorbed in the atmosphere.

At higher energies, the situation changes. For pions of $100 \mathrm{GeV}\left(d_{\pi} \sim 5.6 \mathrm{~km}\right.$, corresponding to a column density of $160 \mathrm{~g} \mathrm{~cm}^{-2}$ measured from the production altitude) the interaction probability starts to dominate over decay. Pions of these energies will therefore produce further tertiary pions in subsequent interactions, which will also decay eventually into muons, but providing muons of lower energy. Therefore, the muon spectrum at high energies is always steeper compared to the parent pion spectrum.

The muon energy and angular distribution is the effect of a convolution of production spectrum, energy losses in the atmosphere and decay. The competition of decay and interaction plays a crucial role and the relative importance of the two processes depends on energy. The mean energy of muons at the ground is about $4 \mathrm{GeV}$ (Nakamura et al., 2010). 
Three different energy regions in the muon spectrum are distinguishable:

- $E_{\mu} \leq \epsilon_{\mu}$, where $\epsilon_{\mu} \sim 1 \mathrm{GeV}$. In this case, muon decay and muon energy loss are important and must be taken into account. Semi-analytical solutions (as those used in the higher energy range) overestimate the flux. The energy spectrum is almost flat, starting to steepen gradually in the same way as the primary spectrum above $10 \mathrm{GeV}$.

- $\epsilon_{\mu} \leq E_{\mu} \leq \epsilon_{\pi, K}$, where $\epsilon_{\pi}=115 \mathrm{GeV}$ and $\epsilon_{K}=850$ $\mathrm{GeV}$ are the critical energies for the vertical directions. Above $\sim 100 \mathrm{GeV}$, the muon flux has the same power law of the parent mesons, and hence of the primary CRs. Below $100 \mathrm{GeV}$, the effect of the muon energy loss in the atmosphere it still important, particularly going towards the horizontal direction.

- $E_{\mu} \gg \epsilon_{\pi, K}$. The meson production spectrum has the same power law dependence of the primary CRs, but the rate of their decay steepens one power of $E_{\mu}$ since the pion and kaon decay probability is suppressed. The thickness of the atmosphere is not large enough for pions to decay, because of the high Lorentz factor. For $E>\epsilon_{\pi}$ the inclined muon spectrum is flatter than the vertical one and the muon flux is respectively higher.

In the intermediate and high energy region (above $100 \mathrm{GeV}$ ) and for zenith angle $\theta<60^{\circ}$ an approximate formula holds:

$$
\frac{d N_{\mu}\left(E_{\mu}, \theta\right)}{d E_{\mu} d \Omega}=A E_{\mu}^{-\gamma}\left(\frac{1}{1+\left(\frac{a E_{\mu}}{\epsilon_{\tau}}\right) \cos \theta}+\frac{B}{1+\left(\frac{b E_{\mu}}{\epsilon_{K}}\right) \cos \theta}\right)
$$

where the scale factor $A$, the power index $\gamma$, the balance factor $B$ (which depends on the ratio of muons produced by kaons and pions), and the $a, b$ coefficients are adjustable parameters. Different best estimates of these parameters were published by several authors. For a review, see Lesparre et al. (2010). Because pions decay more easily in non-vertical showers, a zenith angle $\theta$ factor enters in the formula and muons at large angles have a flatter energy spectrum.

\section{Angular distribution at sea level}

The muon intensity from horizontal directions at low energies is naturally reduced because of muon decays and absorption effects in the thicker atmosphere at large zenith angles. At high energy the parent particles of muons travel relatively long distances in rare parts of the atmosphere. As a consequence, their decay probability is increased compared to the interaction probability.

Figure 2 gives a quantitative description of this effect. Muons below the few $\mathrm{GeV} / \mathrm{c}$ momentum range fade fairly quickly with increasing zenith angle, with dependence $\propto \cos ^{n} \theta$, where $n \sim 2 \div 3$.

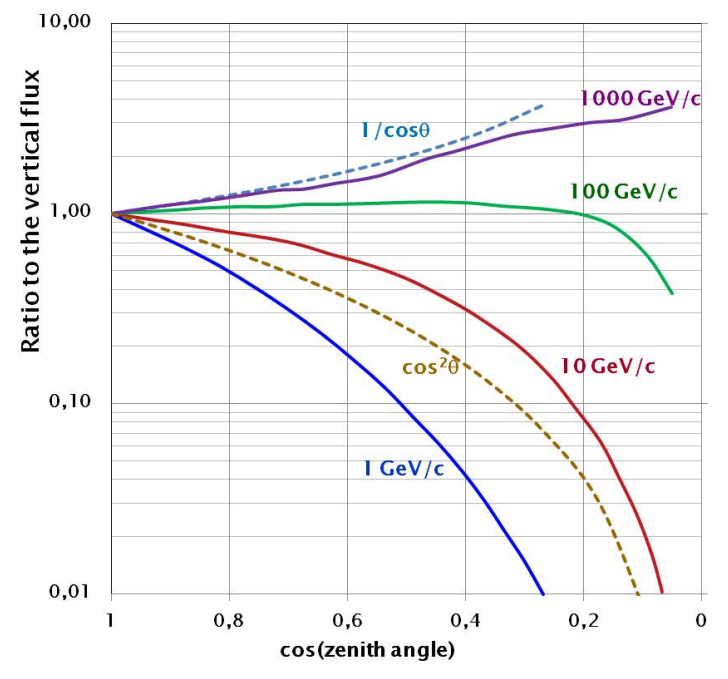

Fig. 2. Angular distribution of muons at the ground for different muon energies. The overall angular distribution of muons measured at sea level is $\alpha \cos ^{2} \theta$, which is characteristic of muons with $E_{\mu} \sim 3 \mathrm{GeV}$. At lower energy the angular distribution becomes increasingly steep, while at higher energy it flattens, approaching a $1 / \cos \theta$ distribution for $E_{\mu} \gg \epsilon_{\pi}$ and $\theta<60^{\circ}$. At large angles low energy muons decay before reaching the surface and high energy pions decay before they interact, thus the average muon energy increases. The estimate of the angular distribution is based on a Monte Carlo and accounts for the curvature of the Earth atmosphere.

The flux of muons in the $100 \mathrm{GeV} / \mathrm{c}$ range is relatively flat up to $\cos \theta \simeq 0.2$ and then quickly declines. At $1 \mathrm{TeV} / \mathrm{c}$ the flux monotonically increases with the zenith angle, approaching the $1 / \cos \theta$ dependence. The flux of $\mathrm{TeV}$ muons is particularly sensitive to large values of the zenith angle. When approaching the horizontal direction, a small difference in $\cos \theta$ changes appreciably the thickness and the density profile of the atmosphere and the corresponding muon energy spectrum. For this reason the measurements of almost horizontal muons is very difficult.

\section{Measurements at sea level}

Measurements performed at ground level offer the advantage of a high stability, large collecting factor and long exposure time due to relatively favourable experimental conditions. Sea level data offer the possibility to perform a robust check of the reliability of existing Monte-Carlo codes.

Many of the experiments devoted to the measurement of the muon momentum spectra and intensity (vertical and inclined directions) have been carried out since the 1970s. The results are often in disagreement with one another; the discrepancies are significantly larger than the experimental reported errors. Recently new instruments, mainly spectrometers designed for balloon experiments or used primarily in 
CERN LEP and LHC experiments and used also to CR studies, have added new valuable information.

The vertical muon intensity at sea level is a quantity that varies with the geomagnetic latitude, altitude, solar activity and atmospheric conditions. When comparing muon observations at low energies $(<20 \mathrm{GeV} / \mathrm{c})$ it is important to know the year and location where the measurements were made (Cecchini and Sioli, 2000).

The geomagnetic field tends to prevent low energy cosmic rays from penetrating through the magnetosphere down to the Earth's atmosphere. At any point on the Earth one can define a threshold (or cut-off) rigidity for cosmic rays arriving at a particular zenith and azimuth angle. Primary nuclei having lower rigidity are deflected by the action of the geomagnetic field and do not produce muons at that latitude. The cut-off values range from less than $1 \mathrm{GV}$ near the geomagnetic poles to about $16 \mathrm{GV}$ for vertical particles near the equator. As CR primaries are predominantly protons and nuclei, it results that at a given location the intensity from the west is stronger than that from the east. The geomagnetic effects are important for sea level muons up to about $E_{\mu} \sim 5 \mathrm{GeV}$, and the effect is larger at higher altitudes.

The 11-yr solar cycle influences the primary CR spectrum at the top of the atmosphere, as the configuration of the interplanetary magnetic field varies. It results that the cosmic ray flux is significantly modulated up to energies of about $20 \mathrm{GeV}$.

Most experiments are not performed exactly at sea level. A correction to take into account the dependence of the flux on the altitude must be included. For muon momenta above 10 $\mathrm{GeV}$ and altitudes $H$ less than $\sim 1000 \mathrm{~m}$, the vertical muon flux can be parameterised as $I_{\mu}(H)=I_{\mu}(0) e^{-H / L(p)}$, where $L(p)=4900+750 p$ is a scale factor (in metres) which depends on muon momentum, $p$ (measured in $\mathrm{GeV} / \mathrm{c}$ ).

Changes in pressure and temperature in the atmosphere above the detector produce variations which will be considered in Sect. 5.2.

\subsection{Experimental setups}

Different experimental methods were used to measure the muon flux and energy spectrum.

Muon telescopes are made of several charged particle detectors arranged along a straight line and interlaid by one or more layers of absorbing material. In some experiments the detector and absorber are in a rigid construction which could be rotated in zenith and azimuth direction, allowing the selection of muons from a given direction of celestial hemisphere. The quantity of material (in $\mathrm{g} \mathrm{cm}^{-2}$ ) travelled by muons in such a telescope is approximately constant and it sets the muon energy threshold.

Multi-directional muon telescopes generally consist of at least two layers of segmented muon detectors. The coincidence of signals between two counters in upper and bottom layers determines the direction of muon arrival. The quantity

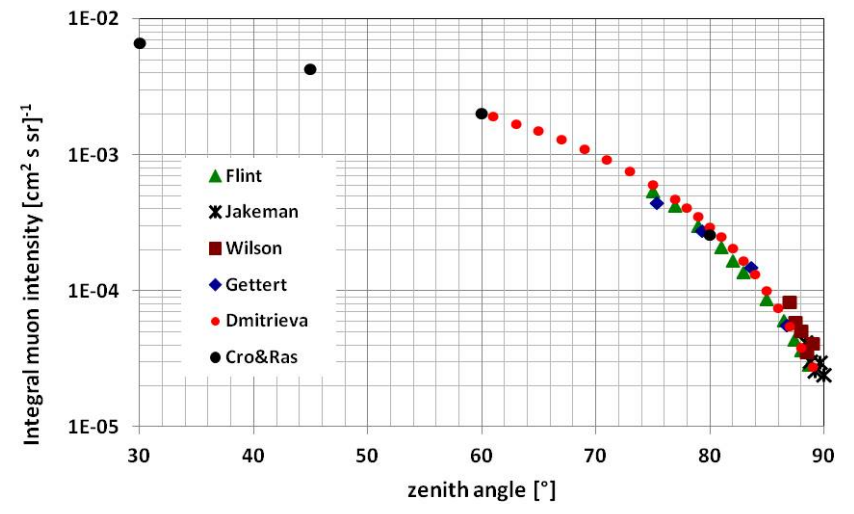

Fig. 3. Variation of absolute integral intensity of muons at sea level with the zenith angle. The momentum threshold $p_{\mathrm{T}}$ is slightly different for each experiment and ranges from 0.1 to $1 \mathrm{GeV} / \mathrm{c}$. The points are normalised assuming $p_{\mathrm{T}}=0.35 \mathrm{GeV} / \mathrm{c}$. The points correspond (from top to bottom): Flint et al. (1972), Jakeman (1956), Wilson (1959), Gettert et al. (1993), Dmitrieva et al. (2006), Crokes and Rastin (1972).

of material crossed by the particle in such detectors increases with increase of zenith angle, so the threshold energy for multidirectional muon telescopes depends on $\theta$. A compilation of measurements of the muon flux at latitudes between 52 and $56^{\circ}$ as a function of the zenith angle by different experiments is reported in Fig. 3.

The muon energy spectrum has been extensively measured, mainly by solid iron magnet spectrometers. For these detectors, the multiple scattering plays an important role in the momentum resolution, particularly at low energies. Recently, measurements have been performed using low mass superconducting magnet spectrometers designed as a balloon-borne apparatus for cosmic ray studies. They represent a sort of second generation experiments.

The atmospheric muon flux and energy spectrum was also measured using the precise muon spectrometer of the L3 detector which was located at the LEP collider at CERN. This apparatus collected muons $\sim 30 \mathrm{~m}$ below a stratified rock overburden, and with dimensions much larger than previous experiments (the volume of the region with a magnetic field of $0.5 \mathrm{~T}$ was $\sim 1000 \mathrm{~m}^{3}$ ).

Finally, information about the muon flux with momenta larger than few $\mathrm{TeV}$ have been extracted from underground measurements, see Sect. 4. Comprehensive review of various types of particle detectors used for cosmic ray studies can be found in Dorman (2004).

\subsection{Momentum spectra at the vertical direction}

Table 1 lists the authors and energy range of the reported absolute vertical intensity measurements. It is also shown if the experiment has been used by other reviews, namely "B" (Bugaev et al., 1998), "H\&T" (Hebbeker and Timmermans, 2002) and in the Particle Data Group "PDG" (Nakamura et 
Table 1. Compilation of different measurements of the muon momentum spectrum. The second column reports the momentum range; the 3rd, 4th and 5th columns if the data are used in the compilation of Bugaev et al. (1998), Hebbeker and Timmermans (2002) (with the used normalisations factor) and Nakamura et al. (2010), respectively.

\begin{tabular}{lrlll}
\hline Reference & $p_{\mu}[\mathrm{GeV} / \mathrm{c}]$ & $\mathrm{B}$ & $\mathrm{H} \& \mathrm{~T}$ & $\mathrm{PDG}$ \\
\hline Ashton and Wolfendale (1963) & $15.1-82.1$ & $\mathrm{X}$ & 0.79 & \\
Ayre et al. (1975) & $20-500$ & $\mathrm{X}$ & & $\mathrm{X}$ \\
Baber et al. (1968) & $11-810$ & $\mathrm{X}$ & $\mathrm{X}$ & \\
Rastin (1984) & $3-3000$ & $\mathrm{X}$ & 0.933 & $\mathrm{X}$ \\
Bateman et al. (1971) & $10-150$ & $\mathrm{X}$ & 0.858 & \\
Allkofer et al. (1968) & $20-1000$ & $\mathrm{X}$ & 1.039 & $\mathrm{X}$ \\
De Pascale et al. (1993)* & $0.25-100$ & $\mathrm{X}$ & 0.944 & $\mathrm{X}$ \\
Kremer et al. (1999)* & $0.2-120$ & & 0.818 & $\mathrm{X}$ \\
Achard et al. (2004) & $20-3000$ & & & $\mathrm{X}$ \\
Haino et al. (2004)* & $0.6-400$ & & & $\mathrm{X}$ \\
\hline
\end{tabular}

* Experiments with magnetic spectrometers.

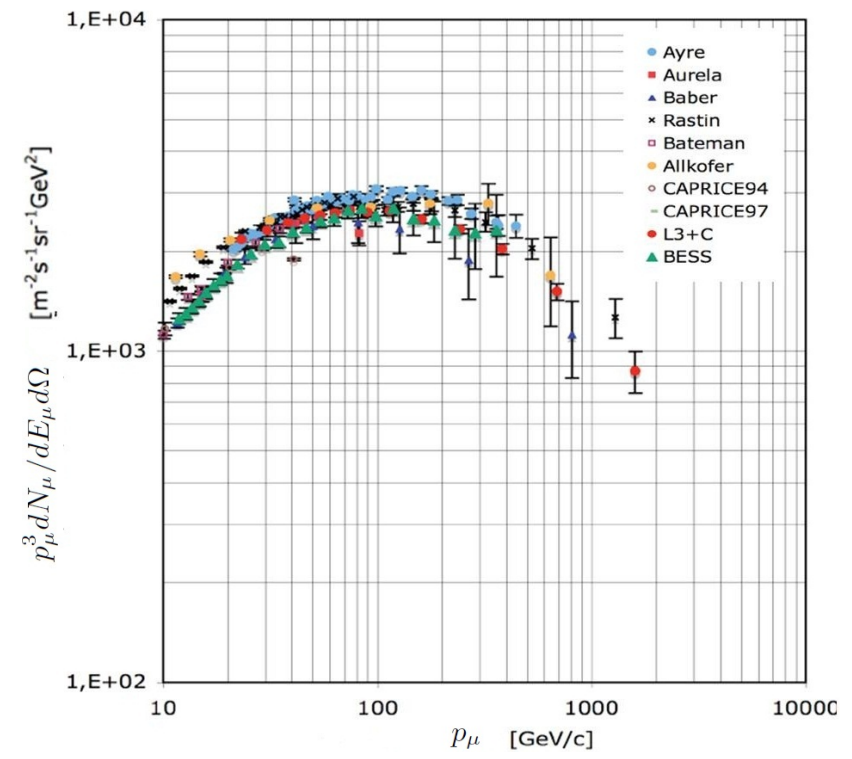

Fig. 4. Vertical absolute differential muon intensity $p_{\mu}^{3} d N_{\mu} / d E_{\mu} d \Omega$ at sea level for the experiments reported in Table 1. The ordinate values have been multiplied by $p_{\mu}^{3}$ in order to compress the plot and to emphasise the differences. In this energy range, $p_{\mu} \simeq E_{\mu}$.

al., 2010). For H\&T we report also the final normalisation factor they have found. The symbol $\left(^{*}\right)$ refers to experiment using superconducting magnet spectrometers.

The measurements listed in Table 1 (only published results) of the muon momentum from the vertical direction are presented in Fig. 4. The agreement between measurements is relatively good and the largest contribution to the deviations are the systematic errors due to incorrect knowledge of the acceptance, efficiency of the counters and corrections for multiple scattering.

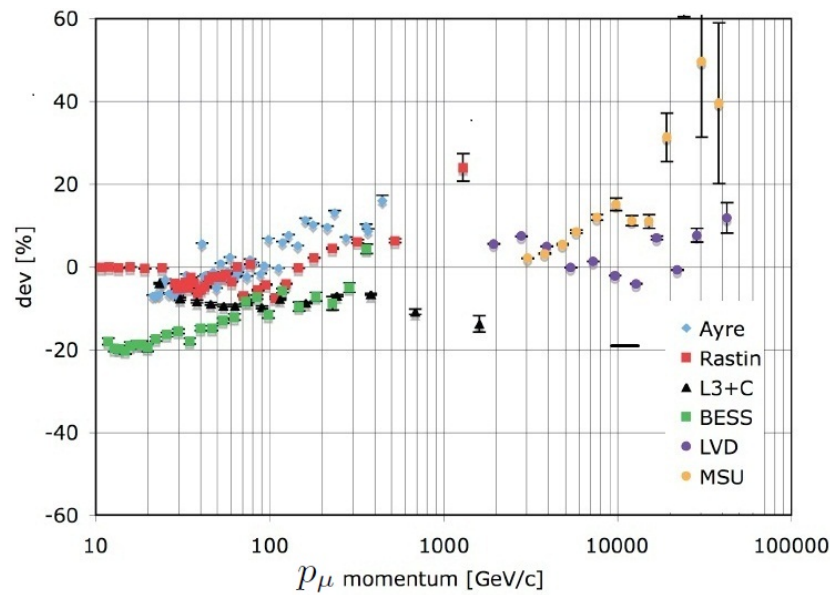

Fig. 5. Relative deviations of the differential muon intensity measurements at sea level with respect to the Bugaev et al. (1998) parameterisation. The symbols are the same as in Fig. 4; the indirect measurements described in Sect. 4.2 and Fig. 7 are also included.

Measurements of the muon momentum spectra for $p_{\mu}<1 \mathrm{TeV} / \mathrm{c}$ are particularly important for the comparison of nuclear cascade models with available data. The differences between the measurements of the sea-level spectra are more evident if the percentage deviations of the data from one of the parameterisation are plotted (see Fig. 5). Differences of $\pm 15-20 \%$ exist between the data and the parameterisation. The disagreement between the different experiments can be as large as 30-35\% despite the fact that the declared individual errors are small (however increasing with momentum due to the decreasing number of detectable particles and to the maximum detectable momentum). The origin of the discrepancies can well be due to the incorrect knowledge/control of systematic effects. For example, in the case of $\mathrm{L} 3+\mathrm{C}$, the stable negative deviations can be due to a bad correction for the molasses that cover the apparatus.

\section{Underground muons}

Underground measurements offer the possibility to extend the energy range of the muon spectrum beyond $1 \mathrm{TeV}$. Such data are of an indirect type, but their link with the direct lower energy observations gives the possibility to complete the picture of muon spectra measurements.

Deep underground detectors have normally large collecting area and are not subject to the time restrictions of balloon and satellite experiments, so they can measure the muon flux for a very long time. As a drawback, as discussed in Sect. 2, the energy dependence of the muon spectrum at $E_{\mu}>1 \mathrm{TeV}$ steepens one power and the intensity is a factor 1000 smaller than that of primary CRs on the upper atmosphere. The penetrating component of CRs underground depends on a complex convolution of different physics processes, as the 
high energy muon production spectrum and the muon energy losses. Particularly important is the knowledge of the composition and of the thickness of the material overburden on top of the detector.

\subsection{Muon energy losses}

Muon energy losses are usually divided into continuous and discrete processes. The former is due to ionisation, which depends weakly on muon energy and can be considered nearly constant for relativistic particles. For muons below $\sim 500 \mathrm{GeV}$, this is the dominant energy loss process. For muons reaching great depths, discrete energy losses become important: bremsstrahlung ("br"), direct electron-positron pair production ("pair") and electromagnetic interaction with nuclei (photo production, "ph"). In these radiative processes energy is lost in bursts along the muon path. In general the total muon energy loss is parameterised as

$\frac{d E_{\mu}}{d X}=-\alpha-\beta E_{\mu}$

where $X$ is the thickness of crossed material in $\mathrm{g} \mathrm{cm}^{-2}$ and $\beta=\beta_{\mathrm{br}}+\beta_{\text {pair }}+\beta_{\mathrm{ph}}$ is the sum of fractional energy loss in the three mentioned radiation processes. As the rock compositions are different for different underground experiments, the so-called standard rock is defined as a common reference. The standard rock is characterised by density $\rho=2.65 \mathrm{~g} \mathrm{~cm}^{-3}$, atomic mass $A=22$ and charge $Z=11$. The thickness $X$ is commonly given in units of metres of water equivalent $\left(1 \mathrm{~m}\right.$ w.e. $\left.=10^{2} \mathrm{~g} \mathrm{~cm}^{-2}\right)$.

The factors $\alpha$ and $\beta$ in Eq. (6) are mildly energy dependent as well as dependent upon the chemical composition of the medium: in particular $\alpha \propto Z / A$ and $\beta \propto Z^{2} / A$. Typical values are $\alpha \simeq 2 \mathrm{MeV} \mathrm{g}^{-1} \mathrm{~cm}^{2}$ and $\beta \simeq 4 \times 10^{-6} \mathrm{~g}^{-1} \mathrm{~cm}^{2}$. The critical energy is defined as the energy at which ionisation energy loss equals radiative energy losses: $\epsilon_{\mu}=\alpha / \beta \simeq 500 \mathrm{GeV}$.

The general solution of Eq. (6) corresponds to the average energy $\left\langle E_{\mu}\right\rangle$ of a beam of muons with initial energy $E_{\mu}^{0}$ after penetrating a depth $X$ :

$$
\left\langle E_{\mu}(X)\right\rangle=\left(E_{\mu}^{0}+\epsilon_{\mu}\right) e^{-\beta X}-\epsilon_{\mu} .
$$

The minimum energy required for a muon at the surface to reach slant depth $X$ is the solution of Eq. (7) with residual energy $E_{\mu}(X)=0$ :

$E_{\mu, \min }^{0}=\epsilon_{\mu}\left(e^{\beta X}-1\right)$.

The range $R$ for a muon of energy $E_{\mu}^{0}$, i.e. the underground depth that this muon will reach, is

$$
R\left(E_{\mu}^{0}\right)=\frac{1}{\beta} \ln \left(1+\frac{E_{\mu}^{0}}{\epsilon_{\mu}}\right) .
$$

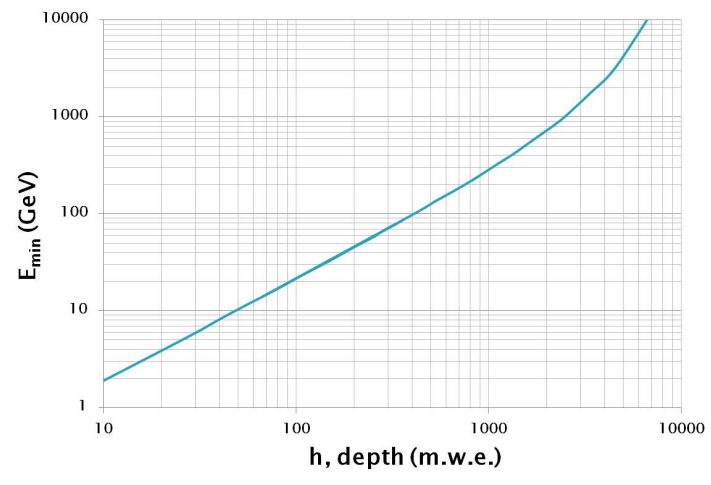

Fig. 6. Minimum energy at surface, $E_{\min }$ to reach a given underground depth $X=h$. Typical values of $E_{\min }$ for muons are 2, 20 and $2000 \mathrm{GeV}$ to reach the ground ( $10 \mathrm{~m}$ w.e.), the LEP tunnel ( $\sim 80$ mw.e.) and the Gran Sasso Laboratory ( $\sim 3 \mathrm{~km}$ w.e. $)$, respectively.

The above quantities are average values. For precise calculations of the underground muon flux one needs to take into account the fluctuations inherent to the radiative processes. Because of the stochastic character of muon interaction processes with large energy transfers (e.g. bremsstrahlung) muons are subject to a considerable range straggling. The higher $E_{\mu}^{0}$ is, the more dominant are the radiation processes and the more important are the fluctuations of the energy losses which broaden the distribution of the range (Lipari and Stanev , 1991). Figure 6 shows the typical values of the minimum energy at surface, $E_{\min }$ to reach a given underground depth.

\subsection{The depth-intensity relation}

The muon spectrum at energies above few $\mathrm{TeV}$ was deduced by underground measurements. The procedure used for this indirect measurement of the sea-level energy spectrum passes through the determination of the so-called depthintensity relation (DIR) $I_{\mu}^{0}(h)$. This quantity represents the muon intensity at the vertical direction as a function of the depth $h$. As underground detectors are at a fixed position, in principle only one point can be measured. However, when measuring the muon intensity $I_{\mu}(h, \theta)$ at different zenith angle $\theta$, the quantity of rock (or water) overburden changes. At high energy $\left(E_{\mu}>1 \mathrm{TeV}\right)$ and for $\theta<60^{\circ}$, Eq. (5) provides a simple relationship between $I_{\mu}^{0}(h)$ and $I_{\mu}(h, \theta)$ :

$I_{\mu}(h, \theta)=I_{\mu}^{0}(h) / \cos \theta$.

From the experimental point of view, $I_{\mu}(h, \theta)$ is measured at a given direction $\theta$, corresponding to a slant depth $h$, as

$$
I_{\mu}(h, \theta)=\left(\frac{1}{\Delta T}\right) \frac{\sum_{i} N_{i} m_{i}}{\sum_{j} \Delta \Omega_{j}(\theta, \phi) A_{j}(\theta, \phi) \epsilon_{j}(\theta, \phi)}
$$

where $\Delta T$ in the total livetime (in s) of the experiment, $N_{i}$ is the number of detected events with multiplicity $m_{i}$ with 


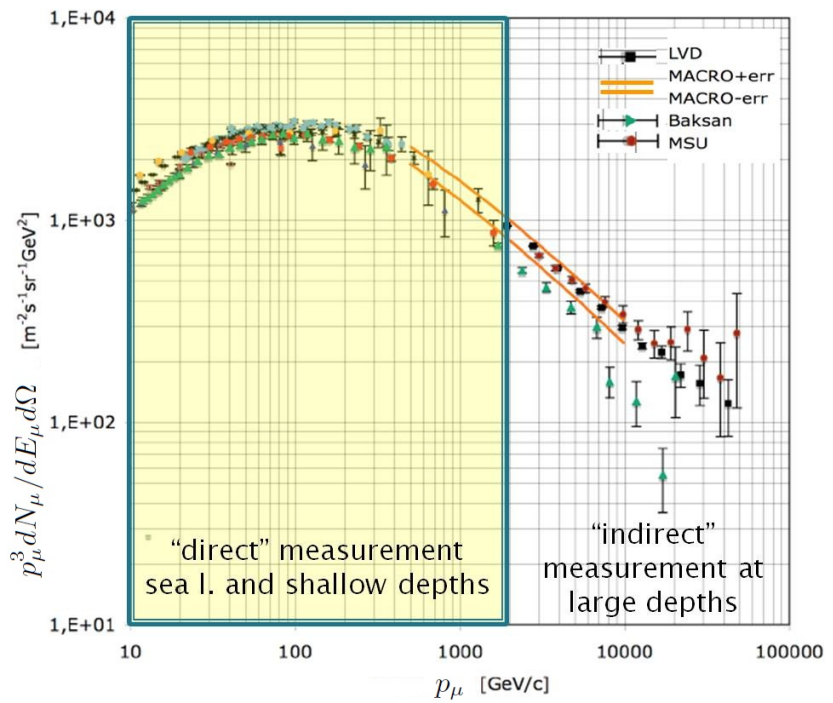

Fig. 7. Extension of the data shown in Fig. 4 to higher momenta, by including the indirect measurement of the underground muon flux.

zenith $\theta$ and azimuth $\phi$ in the angular bin $\Delta \Omega_{j}(\theta, \phi)$ (sr). $A_{j}(\theta, \phi)\left(\mathrm{m}^{2}\right)$ and $\epsilon_{j}(\theta, \phi)$ correspond to the geometrical intrinsic acceptance of the detector, and the overall efficiency in the $(\theta, \phi)$ bin.

The relation between the measured DIR function $I_{\mu}^{0}(h)$ (measured from Eq. 11 with the use Eq. 10) and the differential sea-level muon spectrum $\frac{d N_{\mu}}{d E_{\mu} d \Omega}$ is expressed as

$I_{\mu}^{0}(h)=\int_{0}^{\infty} \frac{d N_{\mu}}{d E_{\mu} d \Omega} P\left(E_{\mu}, h\right) d E_{\mu}$.

Here, $P\left(E_{\mu}, h\right)$ is the muon survival function. It represents the probability that muons of energy $E_{\mu}$ at surface reach a given depth $h$, and it is determined via Monte Carlo calculations. Assuming Eq. (5) for the sea-level muon spectrum, leaving as free parameters the muon spectral index $\gamma$, the scale $A$ and the balance $B$ factors, it is possible to unfold the sea level muon spectrum from the measured vertical muon intensity.

Table 2 indicates the experiments, the depth and the estimated muon momentum range for these indirect underground measurements. The results are shown in Fig. 7. The differential distribution $\left(d N_{\mu} / d E_{\mu} d \Omega\right)$ is presented, as usual, multiplied by a factor $p_{\mu}^{3}$ (as momentum and energy coincide) to better observe the variation of the spectrum in the whole energy region. In these indirect measurements, the main sources of systematic uncertainties are due to the treatment of hard processes in the energy loss of muons and to the knowledge of the rock density and overburden thickness, which rely on geological surveys.

This situation seems to favour underwater/ice experiments. In this case, the uncertainty in the material density and overburden is negligible with respect to underground
Table 2. Compilation of different indirect measurements of the muon momentum with underground detectors.

\begin{tabular}{llr}
\hline $\begin{array}{l}\text { Experiment } \\
\text { (Reference) }\end{array}$ & $\begin{array}{l}\text { Depth } \\
(\mathrm{m} w . e .)\end{array}$ & $\begin{array}{r}\text { Momentum } \\
(\mathrm{TeV} / \mathrm{c})\end{array}$ \\
\hline LVD (Aglietta et al., 1995) & $>3000$ & $1.9-43$ \\
MACRO (Ambrosio et al., 1995) & $>3150$ & $0.5-20$ \\
Baksan (Bakatanov et al., 1992) & $>850$ & $1-30$ \\
MSU (Zatsepin et al., 1994) & $>50$ & $3-50$ \\
\hline
\end{tabular}

experiments. However, when small-size experiments are carried out, the main uncertainty is the multiplicity of the muon bundle. In the case of large underwater experiments (like the running neutrino telescopes, Chiarusi and Spurio, 2010), they are optimised to look for upward-going neutrino induced particles. Atmospheric muons are seen with the "tail of the eyes" (the photomultipliers inside optical modules), where large uncertainties on the optical module angular acceptance do not allow a precision measurements. See Aguilar et al. (2010) for a discussion and the DIR measurement with the ANTARES underwater neutrino telescope.

\section{Characteristic of underground/underwater muons}

Figure 8 shows a multiple muon event detected by MACRO. Multiple events are closely packed bundles of parallel muons, usually of high energy, originating from the same primary CR. Multi-muon events are used to explore the properties of very high energy hadronic interactions and to study the longitudinal development of showers (Ambrosio et al., 1997a,b). The multiplicity of produced secondary particles increases with the energy of the initiating particle. The muon multiplicity is an observable which is also correlated with the mass of the primary CR: at a given total energy, heavier nuclei produce more muons than a primary proton.

The interaction vertex of the particles which initiate the air showers is typically at an atmospheric altitude of $15 \mathrm{~km}$. Since secondary particles in hadronic cascades have small transverse momenta $p_{\mathrm{t}}(\sim 300 \mathrm{MeV} / \mathrm{c})$, high energy muons are essentially collimated near the shower axis. Considering a primary nucleon, producing mesons of energy $E_{\pi, K}$ with transverse momentum $p_{\mathrm{t}}$ at a height $H_{\text {prod }}$, the average separation of their daughter high energy muons from the shower axis is given by Ambrosio et al. (1999):

$r \simeq \frac{p_{\mathrm{t}}}{E_{\pi, K}} H_{\text {prod }}$.

For primary energies around $10^{14} \mathrm{eV}$, the lateral displacements of energetic muons $(\sim 1 \mathrm{TeV})$ of several metres are typically obtained underground. Displacements are almost exclusively caused by transferred transverse momentum in hadronic processes. Typical multiple scattering angles for 


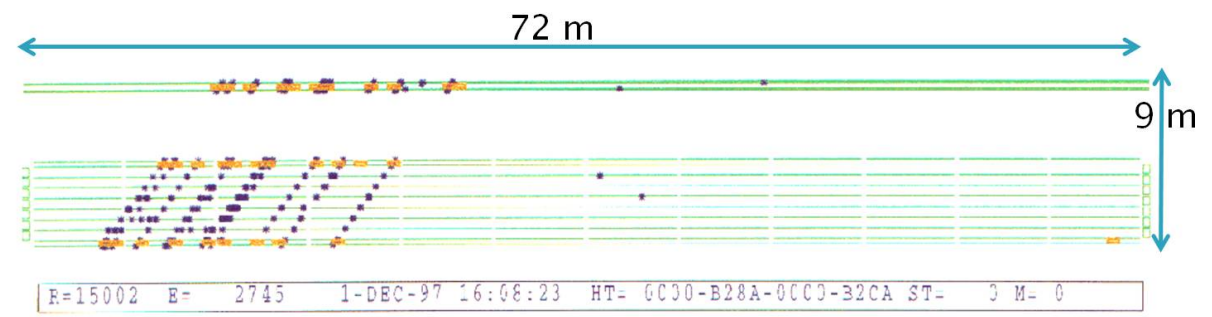

Fig. 8. A multimuon event seen in the MACRO experiment. 10 different tracks are identified.

muon energies around $100 \mathrm{GeV}$ in thick layers of rock (50$100 \mathrm{~m}$ ) are of the order of a few mrad.

\subsection{Atmospheric muons in neutrino telescopes}

Atmospheric muons represent the most abundant signal in a neutrino telescope and can be used to calibrate the detector and to check its expected response to the passage of charged particles. On the other side, they can represent a dangerous background source because downward-going muons can incorrectly be reconstructed as upward-going particles and mimic high energy neutrino interactions; muons in bundles are particularly dangerous. These muons are expected to arrive almost at the same time in the plane perpendicular to the shower axis. A full Monte Carlo simulation, starting from the simulation of atmospheric showers, can accurately reproduce the main features of muons reaching a neutrino telescope, but requires a large amount of $\mathrm{CPU}$ time.

Recently, parametric formulae (Becherini et al., 2006) to evaluate the flux of atmospheric muons were derived from a full Monte Carlo simulation. These formulas take into account the muon multiplicity and the energy spectrum of muons in a bundle, as a function of the distance from the shower axis. A simple generator interface is provided (called MUPAGE), which can be used by all experiments having a flat overburden coverage of at least $1500 \mathrm{~m}$ w.e. (Carminati et al., 2008). MUPAGE is used to simulate atmospheric muons in Mediterranean neutrino telescopes.

The flux - which corresponds to the $\left(d N_{\mu} / d E_{\mu} d \Omega\right)$ of Eq. (5) integrated over the muon energy $E_{\mu}$, with units: $\mathrm{m}^{-2} \mathrm{~s}^{-1} \mathrm{sr}^{-1}$ - of muon bundles with multiplicity $m$ (see Fig. 9) is obtained as a function of the depth along the vertical direction $h_{0}$ (note that the index 0 means that the depth $h$ is computed exactly at the vertical direction) and zenith angle $\theta$ as

$\Phi\left(m ; h_{0}, \theta\right)=\frac{K\left(h_{0}, \theta\right)}{m^{v\left(h_{0}, \theta\right)}}$.

The flux of bundles of increasing multiplicity $m$ decreases with increasing vertical depth and zenith angle. The parametric dependences of $K\left(h_{0}, \theta\right)$ and $v\left(h_{0}, \theta\right)$ are reported in Becherini et al. (2006), as the others below.

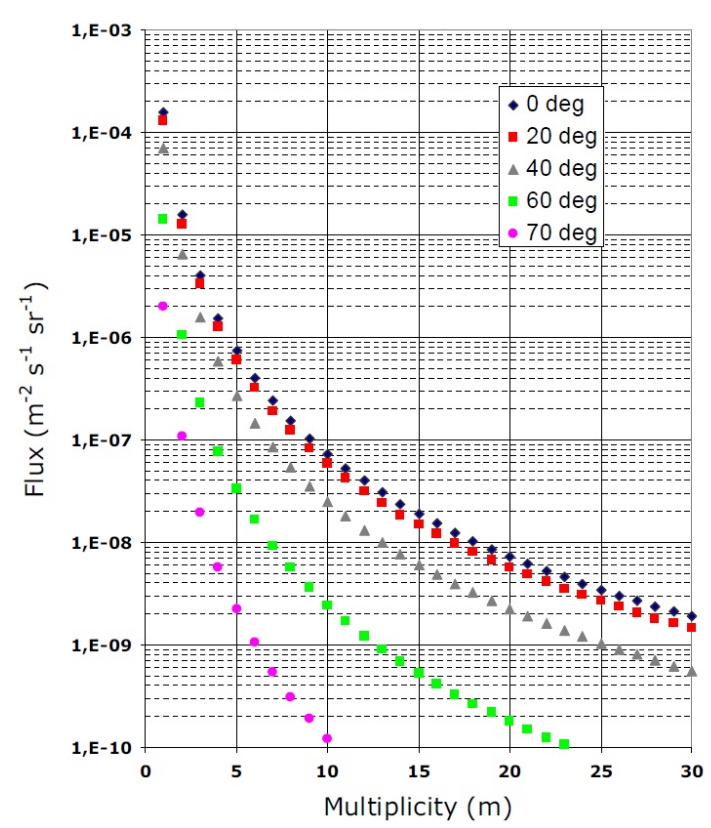

Fig. 9. Flux of bundles of muons as a function of their multiplicity $m$ in the bundle obtained with Eq. (14) at the depth of $3 \mathrm{~km}$ w.e. and for 5 different values of the zenith angle. The computation assumes here a muon energy threshold of $1 \mathrm{GeV}$.

The energy spectrum of muons is described by Gaisser (1990):

$\frac{d N}{d\left(\log _{10} E_{\mu}\right)}=G E_{\mu} e^{\beta X(1-\gamma)}\left[E_{\mu}+\epsilon\left(1-e^{-\beta X}\right)\right]^{-\gamma}$

where $X=h_{0} / \cos \theta, \gamma$ is the spectral index of the primary CRs and $\epsilon=\alpha / \beta ; \alpha, \beta$ are defined in Eq. (6). In the parameterisation, $\gamma$ and $\epsilon$ were instead considered as free fit parameters. The constant $G=G(\gamma, \epsilon)$ represents a normalisation factor, in order that the integral over the muon energy spectrum (Eq. 15) from $1 \mathrm{GeV}$ to $500 \mathrm{TeV}$ is equal to 1 . In the case of single muon events (bundles with $m=1$ ) a simple dependence $\gamma=\gamma\left(h_{0}\right)$ and $\epsilon=\epsilon\left(h_{0}, \theta\right)$ holds.

The situation is more complicated for multiple muons. Due to the muon production kinematics, the muon energy depends on their distance with respect to the axis of the bundle. The description of the muon lateral distance $R$ from the axis is thus the preliminary step to evaluate the muon energy 


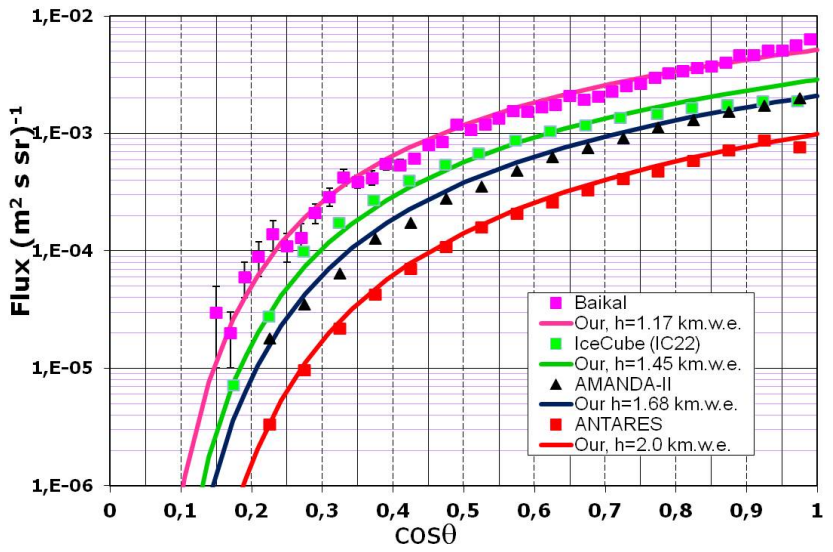

Fig. 10. Muon flux as a function of the $\cos \theta$ as measured by ANTARES (Aguilar et al., 2010), AMANDA-II (Desiati et al., 2003), IceCube (Berghaus, 2009), and Baikal (Belolaptikov et al., 1997) at four different depths. The results of the MUPAGE computation is superimposed as full lines.

distribution in a bundle. $R$ (in the plane orthogonal to the axis) was extracted from a distribution of the form:

$\frac{d N}{d R}=C \frac{R}{\left(R+R_{0}\right)^{\alpha}}$

where $R_{0}=R_{0}\left(h_{0}, m, \theta\right)$ and $\alpha=\alpha\left(h_{0}, m\right)$. The energy spectrum of muons arriving in bundles has the same general form as for single muons (Eq. 15). In the case of multiple muons, the analytic description of the parameters $\gamma=\gamma(h, R, m)$ and $\epsilon=\epsilon(h, R, \theta)$ depends on 15 constants.

These parameterisations allow to evaluate not only the total muon flux, but also the total number of muon bundles in deep detectors starting from the primary CR flux, CR composition and interaction model which reproduces (at the level of 30\%) the MACRO data (depth: 3000-6000 $\mathrm{m}$ w.e., $\left.\theta<60^{\circ}\right)$. Figure 10 shows the comparisons of the zenith distribution evaluated at a fixed depth using Eq. (14) and some underwater/ice data.

\subsection{Pressure and temperature effect}

If atmospheric temperature changes by $\Delta T$, the muon flux at the observation level $X$ will change by a quantity $\Delta I_{\mu}$. This variation can be either positive or negative, i.e. $\Delta I_{\mu} \propto \pm \Delta T$, depending on the energy $E_{\mu}$ of the observed muon. Two competitive effects are in action if the atmospheric temperature increases. The atmosphere expands and the air density decreases: the probability of the interaction of mesons (kaons and pions) at unit of geometric path becomes smaller, hence decay probability into muons becomes higher. On the other hand, the geometric expansion of the atmosphere increases the path from the generation point to the detector, and a higher number of muons will decay. For low energy muons, the latter effect is the dominant one, and the correlation sign between flux and temperature is negative

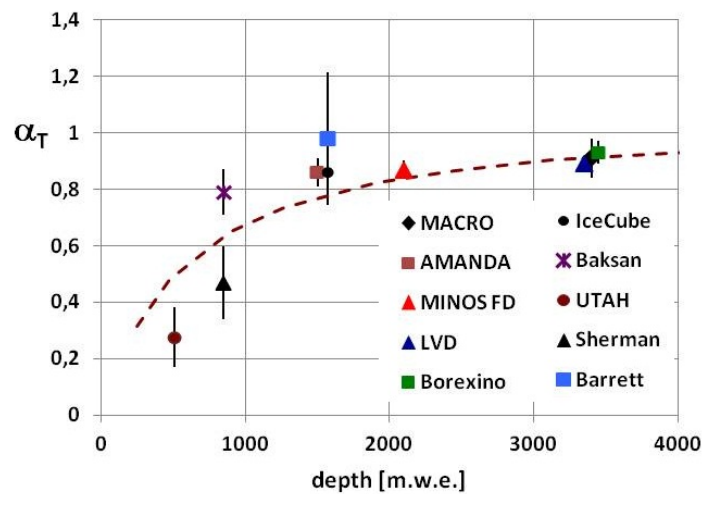

Fig. 11. The temperature coefficient $\alpha_{T}$ as a function of detector depth. The dashed curve is the prediction using the pion-only model (Ambrosio et al., 1997c). The points correspond (from top to bottom): Ambrosio et al. (1997c), Bouchta (1999), Adamson et al. (2010), Selvi (2009), Bellini (2012), Desiati (2011), Andreyev et al. (1991), Cutler et al. (1981), Sherman (1954), Barrett et al. (1954).

$\left(\Delta I_{\mu} \propto-\Delta T\right)$. For high energies, muons have not enough time to decay in the atmosphere and the correlation sign becomes positive (Dmitrieva et al., 2011).

Many underground/water/ice experiments measured the correlation between temperature and high energy muon intensity. It was found that Ambrosio et al. (1997c) an effective temperature $T_{\text {eff }}$, defined by the weighted average of temperatures from the surface to the top of the atmosphere, is useful to describe the situation. $T_{\text {eff }}$ approximates the atmosphere as an isothermal body, weighting each pressure layer according to its relevance to muon production in atmosphere. The variation of muon rate $\Delta I_{\mu} / I_{\mu}$ is related to the effective temperature as

$\frac{\Delta I_{\mu}}{I_{\mu}}=\alpha_{T} \frac{\Delta T_{\mathrm{eff}}}{T_{\mathrm{eff}}}$

where $\alpha_{T}$ is the atmospheric temperature coefficient, which is a function of both the muon threshold energy and the $K / \pi$ ratio. As the energy increases, the muon intensity becomes more dependent on the meson critical energy $\epsilon_{\pi, K}$, which in turn depends on the atmospheric temperature (Grashorn et al., 2010). The $\alpha_{T}$ coefficient reflects the fraction of mesons that are sensitive to atmospheric temperature variations. For energies much greater than the critical energy, and thus for very deep experiments, the value of $\alpha_{T}$ approaches unity. The expected effective temperature coefficient as a function of depth is shown in Fig. 11, together with the values measured by underground/ice experiments.

\section{Muons and neutrinos}

As indicated in Eq. (1a), the production mechanisms of atmospheric muons and neutrinos are strongly correlated, see Illana et al. (2011) for a recent review. However, due to 


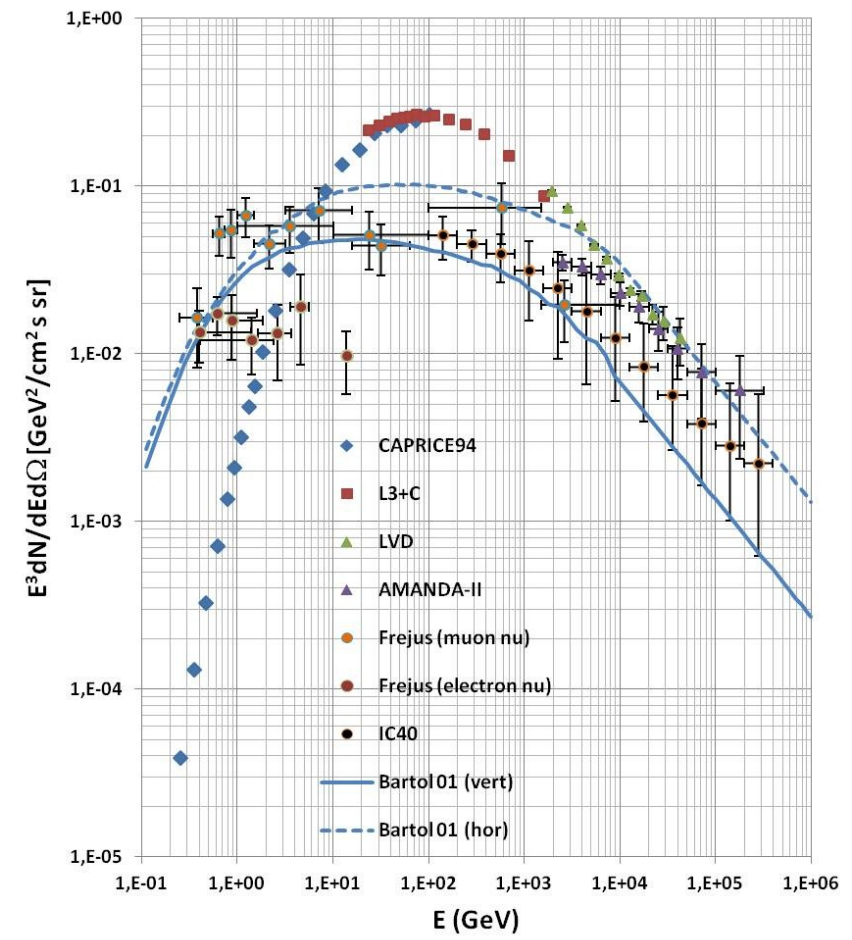

Fig. 12. Compilation of measurements of atmospheric muons and neutrinos. The points correspond (from top to bottom): De Pascale et al. (1993), Achard et al. (2004), Aglietta et al. (1995), Abbasi et al. (2009), Daum et al. (1995), Abbasi et al. (2011). The lines represent the neutrino flux expectation (full: vertical direction; dashed: horizontal direction) computed by Barr et al. (2004).

the two-body kinematics, the energy spectra of the $\mu$ 's and $v_{\mu}$ 's from mesons decay are different. Let us consider for instance the pion decay in the centre of mass (c.m.) system $\left(m_{\pi}=139.6 \mathrm{GeV} ; m_{\mu}=105.7 \mathrm{GeV}\right)$. The c.m. muon energy is $E_{\mu}^{*}=\left(m_{\pi}^{2}+m_{\mu}^{2}\right) / 2 m_{\pi}=109.8 \mathrm{MeV}$. Similarly for the neutrino, considering that in the c.m. system $E_{\mu}^{*}+E_{\nu}^{*}=m_{\pi}$, one has: $E_{v}^{*}=\left(m_{\pi}^{2}-m_{\mu}^{2}\right) / 2 m_{\pi}=29.8 \mathrm{MeV}$. In the laboratory system, the energies are boosted by the Lorentz factor $\gamma=E_{\pi} / m_{\pi}$. In any case, muons carry a larger fraction of the meson energy than neutrinos. As a consequence, the energy distribution of $v_{\mu}$ is slightly shifted towards lower energy values than charged muons, as shown in Fig. 12. Additional $v_{\mu}$ are produced by the in flight decay of muons, together with a $v_{\mathrm{e}}$ and an electron/positron. As the muon decay probability in the atmosphere decreases with increasing $E_{\mu}$, the $v_{\mathrm{e}}$ spectrum is depleted with respect to that of $v_{\mu}$ at high energy. In Fig. 12, we include the measurement of the $v_{\mu}$ energy spectrum reported by the Frejus, AMANDA and IceCube experiments. The $v_{\mathrm{e}}$ component was measured between $\left\langle E_{\mu}\right\rangle \sim 0.4-14 \mathrm{GeV}$ by the Frejus experiment. The measurement of the muon and neutrino energy spectra represents a very challenging result, as completely different experimental techniques were used to measure the charged and neutral leptons in different energy ranges.
Neutrino telescopes are taking data under the Antarctic ice, under the Baikal lake and in the Mediterranean sea. The main goals are the detection of neutrinos from cosmic sources and the measurement of the isotropic flux of high energy neutrinos from the ensemble of all extragalactic sources. The signature for the former is an excess of events over the background of atmospheric neutrinos from a given direction. The signatures of the diffuse astrophysical neutrino signal are (a) isotropy; (b) a hard energy spectrum; (c) approximately equal fluxes of $v_{\mathrm{e}}, v_{\mu}$ and $v_{\tau}$. The neutrino fluxes generated by the prompt charm decay have also the properties (a) and (b), and equal fluxes for $v_{\mathrm{e}}$ and $v_{\mu}$ and therefore constitute a dangerous background.

\section{Conclusions}

The energy spectrum of atmospheric muons is measured over almost 6 decades of muon momentum with different experimental techniques at sea level and in underground/water/ice experiments. The so-called prompt component, which is expected to dominate the spectrum at very high energies, is still undetected. Below $1 \mathrm{TeV} / \mathrm{c}$, disagreements between different experiments are up to $20 \%$ due to systematic uncertainties. Slightly larger uncertainties arise from the indirect methods used to deconvolve the higher energy spectrum up to $\sim 40 \mathrm{TeV}$. These measurements of the energy spectrum above few $\mathrm{TeV}$ were made with large underground detectors. In particular, the MACRO experiment at the Gran Sasso Laboratory in Italy accurately studied the multiplicity distribution of muons arriving in bundle, their lateral distribution with respect to the shower axis and their energy spectrum. These data were used to obtain a parameterisation of the atmospheric muons detected by large underwater neutrino telescopes.

The energy spectrum of atmospheric muon neutrinos are measured through the detection of upgoing muons, generated by charged current interactions of atmospheric $v_{\mu}$. They represent the irreducible background for searches of cosmic neutrinos, and for this reason this component must be accurately known. The $v_{\mathrm{e}}$ component has still to be accurately measured, particularly in the high energy region.

The knowledge of the atmospheric muon spectrum, the characteristics of the muon flux at sea level and the processes of attenuation of muons passing through rocks or other materials is of fundamental importance for Earth science purposes and muon imaging feasibility.

Acknowledgements. M. S. thanks the conference organizers for the invitation at the Muon and Neutrino Radiography 2012 (MNR 2012) hold in Clermont Ferrand. The authors would acknowledge the collaboration of the Bologna colleagues of the (former) MACRO experiment, and of the Opera and ANTARES collaborations. 
Edited by: C. Carloganu

\section{References}

Abbasi, R. et al. - IceCube Coll.: Determination of the atmospheric neutrino flux and searches for new physics with AMANDAII, Phys. Rev., D79, 102005, doi:10.1103/PhysRevD.79.102005, 2009.

Abbasi, R. et al. - IceCube Coll.: Measurement of the atmospheric neutrino energy spectrum from $100 \mathrm{GeV}$ to $400 \mathrm{TeV}$ with IceCube, Phys. Rev., D83, 012001, doi:10.1103/PhysRevD.83.012001, 2011.

Achard, P. et al. - L3 + C Coll.: Measurement of the atmospheric muon spectrum from 20 to $3000 \mathrm{GeV}$, Phys. Lett., B598, 15, doi:10.1016/j.physletb.2004.08.003, 2004.

Adamson, P. et al. - MINOS Coll.: Observation of muon intensity variations by season with the MINOS far detector, Phys. Rev., D81, 012001, doi:10.1103/PhysRevD.81.012001, 2010.

Aglietta, M. et al. - LVD Coll.: Muon depth - intensity relation measured by LVD underground experiment and cosmicray muon spectrum at sea level, Phys. Rev., D58, 092005, doi:10.1103/PhysRevD.58.092005, 1998.

Aguilar, J. A. et al. - ANTARES Coll.: Zenith distribution and flux of atmospheric muons measured with the 5-line ANTARES detector, Astropart. Phys., 34, 179, doi:10.1016/j.astropartphys.2010.07.001, 2010.

Allkofer, O. C., Carstensen, K., and Dau, W. D.: The absolute cosmic ray muon spectrum at sea level, Phys. Lett. B, 36, 425, doi:10.1016/0370-2693(71)90741-6, 1971.

Ambrosio, M. et al. - MACRO Coll.: Vertical muon intensity measured with MACRO at the Gran Sasso Laboratory, Phys. Rev., D52, 3793, doi:10.1103/PhysRevD.52.3793, 1995.

Ambrosio, M. et al. - MACRO Coll.: High-energy cosmic ray physics with the MACRO detector at Gran Sasso: Part 1. Analysis methods and experimental results, Phys. Rev., D56, 1407, doi:10.1103/PhysRevD.56.1407, 1997a.

Ambrosio, M. et al. - MACRO Coll.: High-energy cosmic ray physics with the MACRO detector at Gran Sasso: Part 2. Primary spectra and composition, Phys. Rev., D56, 1418, doi:10.1103/PhysRevD.56.1418, 1997b.

Ambrosio, M. et al. - MACRO Coll.: Seasonal variations in the underground muon intensity as seen by MACRO, Astropart. Phys., 7, 109, doi:10.1016/S0927-6505(97)00011-X, 1997c.

Ambrosio, M. et al. - MACRO Coll.: High statistics measurement of the underground muon pair separation at Gran Sasso, Phys. Rev., D60, 032001, doi:10.1103/PhysRevD.60.032001, 1999.

Andreyev, Yu. M., Chudakov, A. E., Kozyarivsky, V. A., Poddubny, V. Ya., and Tulupova, T. I.: Season behaviour of the diurnal intensity of muons with $E_{\mu}>220$, Proc. 22nd ICRC, Dublin, 3, 693, 1991.

Ashton, F. and Wolfendale, A. W.: The momentum spectrum of cosmic ray muons at an angle of $80^{\circ}$ to the zenith at sea level, Proc. Phys. Soc. Lond., 81, 593, doi:10.1088/0370-1328/81/4/301, 1963.

Ayre, C. A., Baxendale, J. M., Hume, C. J., Nandi, B. C., Thompson, M. G., and Whalley, M. R.: Precise measurement of the vertical muon spectrum in the range $20 \mathrm{GeV} / \mathrm{c}-500 \mathrm{GeV} / \mathrm{c}$, J. Phys., G1, 584, doi:10.1088/0305-4616/1/5/010, 1975.
Baber, S. R., Nash, W. F., and Rastin, B. C.: The momentum spectrum of muons at sea-level in the range $3-1000 \mathrm{GeV} / \mathrm{c}$, Nucl. Phys., B4, 539, doi:10.1016/0550-3213(68)90112-0, 1968.

Bakatanov, V. N., Novoseltsev, Yu. F., Novoseltseva, R. V., Semenov, A. M., and Chudakov, A. E.: Intensity of cosmic ray muons and of primary nucleons according to data from the Baksan underground scintillation telescope, Sov. J. Nucl. Phys., 55, 1169-1174, 1992.

Barr, G. D., Gaisser, T. K., Lipari, P., Robbins, S., and Stanev, T.: Three-dimensional calculation of atmospheric neutrinos, Phys. Rev., 70, 023006, doi:10.1103/PhysRevD.70.023006, 2004.

Barrett, P. H., Cocconi, G., Eisenberg, Y., and Greisen, K.: Diurnal variations of the intensity of cosmic rays far underground, Phys. Rev., 95, 1571, doi:10.1103/PhysRev.95.1571, 1954.

Bateman, B. J., Cantrell, W. G., Durda, D. R., Duller, N. M., Green, P. J., Jelinek, A. V., Nagy, T. A., and Sheldon, W. R.: Absolute measurement of the vertical cosmic ray muon intensity at 3-50 GeV/c near sea level, Phys. Lett., B36, 144, doi:10.1016/0370-2693(71)90130-4, 1971.

Becherini, Y., Margiotta, A., Sioli, M., and Spurio, M.: A Parameterisation of single and multiple muons in the deep water or ice, Astropart. Phys., 25, 1, doi:10.1016/j.astropartphys.2005.10.005, 2006.

Bellini, G. et al. - Borexino Coll.: Cosmic-muon flux and annual modulation in Borexino at $3800 \mathrm{~m}$ water-equivalent depth, J. Cosmol. Astropart. Phys., 05, 015, doi:10.1088/14757516/2012/05/015, 2012.

Belolaptikov, I. A. et al. - Baikal Coll.: The Baikal underwater neutrino telescope: Design, performance, and first results, Astropart. Phys., 7, 263, doi:10.1016/S0927-6505(97)00022-4, 1997.

Berghaus, P. - for the IceCube Coll.: Muons in IceCube, Nucl. Phys. Proc. Suppl., 196, 261, doi:10.1016/j.nuclphysbps.2009.09.050, 2009.

Bouchta, A. - AMANDA Coll.: Seasonal variation of the muon flux seen by AMANDA, Proc. 26th ICRC, HE 3.1.06, Salt Lake City, USA, 2, 108-111, 1999.

Braibant, S., Giacomelli, G., and Spurio, M.: Particles and Fundamental Interactions, Springer, 2012.

Bugaev, E. V., Misaki, A., Naumov, V. A., Sinegovskaya, T. S., Sinegovsky, S. I., and Takahashi, N.: Atmospheric muon flux at sea level, underground, and underwater, Phys. Rev. D, 58, 054001, doi:10.1103/PhysRevD.58.054001, 1998.

Carminati, G., Margiotta, A., and Spurio, M.: Atmospheric MUons from PArametric formulas: a fast GEnerator for neutrino telescopes (MUPAGE), Comput. Phys. Commun., 179, 915, doi:10.1016/j.cpc.2008.07.014, 2008.

Cecchini S. and Sioli M.: Cosmic ray muon physics, Trieste, Italy, 29 June-10 July 1998, 18 pp., 2000.

Chiarusi, T. and Spurio, M.: High-energy astrophysics with neutrino telescopes, Eur. Phys. J., C65, 649, doi:10.1140/epjc/s10052009-1230-9, 2010.

Crokes, J. N. and Rastin, B. C.: The absolute intensity of muons at $31.6 \mathrm{hg} \mathrm{cm}^{-2}$ below sea-level, Nucl. Phys., B39, 493, doi:10.1016/0550-3213(73)90549-X, 1972.

Cutler, D. J. et al. - The Utah Group: Meteorological effects in cosmic ray productions, Proc. 17th ICRC, Paris, France, 4, 290, 1981. 
Daum, K. et al. - Frejus Coll.: Determination of the atmospheric neutrino spectra with the Frejus detector, Z. Physik C, 66, 417, doi:10.1007/BF01556368, 1995.

De Pascale, M. P. et al. - CAPRICE Coll.: Absolute spectrum and charge ratio of cosmic ray muons in the energy region from $0.2 \mathrm{GeV}$ to $100 \mathrm{GeV}$ at $600 \mathrm{~m}$ above sea level, J. Geophys. Res., 98, 3501, doi:10.1029/92JA02672, 1993.

Desiati, P. - IceCube Coll.: Energy dependence of the large scale galactic cosmic rays anisotropy measured with IceCube, Proc. 32nd ICRC, Beijing, 1, 78, 2011.

Desiati, P. et al. - AMANDA Coll.: Response of AMANDA-II to cosmic ray muons, Proc. 28th ICRC, HE 2.3, Tsukuba, 1373, 2003.

Dmitrieva, A. N., Kokoulin, R. P., Kompaniets, K. G., Petrukhin, A. A., Saavedra, O., Timashkov, D. A., Chernov, D. V., Shutenko, V. V., and Yashin, I. I.: Measurements of integral muon intensity at large zenith angles, Phys. Atom. Nucl., 69, 865, doi:10.1134/S1063778806050097, 2006.

Dmitrieva, A. N., Kokoulin, R. P., Petrukhin, A. A., and Timashkov, D. A.: Corrections for temperature effect for ground-based muon hodoscopes, Astropart. Phys., 34, 401, doi:10.1016/j.astropartphys.2010.10.013, 2011.

Dorman, L. I.: Cosmic Rays in the Earth's Atmosphere and Underground, Kluwer Academic Publisher, 2004.

Flint, R. W., Hicks, R. B., and Standil, S.: Variation with zenith angle of the integral intensity of muons near sea level, Can. J. Phys., 50, 843, doi:10.1139/p72-118, 1972.

Gaisser, T. K.: Cosmic Rays and Particle Physics, Cambridge University Press, Cambridge, 1990.

Gaisser, T. K.: Semi-analytic approximations for production of atmospheric muons and neutrinos, Astropart. Phys., 16, 285, doi:10.1016/S0927-6505(01)00143-8, 2002.

Gettert, M., Unger, J., Trezeciak, R., Engler, J., and Knapp, J.: The momentum spectrum of horizontal muons up to $15 \mathrm{TeV} / \mathrm{c}$, Proc. 23rd ICRC, Calgary, 4, 394, 1993.

Grashorn, E. W., de Jong, J. K., Goodman, M. C., Habig, A., Marshak, M. L., Mufson, S., Osprey, S., and Schreiner, P.: The atmospheric charged kaon/pion ratio using seasonal variation methods, Astropart. Phys., 33, 140, doi:10.1016/j.astropartphys.2009.12.006, 2010.

Grieder, P. K. F.: Extensive Air Showers, Springer-Verlag, Berlin, 2010.

Haino, S. et al. - BESS-TeV Coll.: Measurements of primary and atmospheric cosmic-ray spectra with the BESS-TeV spectrometer, Phys. Lett., B594, 35, doi:10.1016/j.physletb.2004.05.019, 2004.
Hebbeker, T. and Timmermans, C.: A compilation of high energy atmospheric muon data at sea level, Astropart. Phys., 18, 107, doi:10.1016/S0927-6505(01)00180-3, 2002.

Illana, J. I., Lipari, P., Masip, M., and Meloni, D.: Atmospheric lepton fluxes at very high energy, Astropart. Phys. 34, 663, doi:10.1016/j.astropartphys.2011.01.001, 2011.

Jakeman, D.: Production of cosmic ray mesons at large zenith angles, Can J. Phys., 34, 432, doi:10.1139/p56-052, 1956.

Kremer, J., Boezio, M., Ambriola, M. L., Barbiellini, G., Bartalucci, S., Bellotti, R., Bergström, D., Bravar, U., Cafagna, F., Carlson, P., Casolino, M. Castellano, M., Ciacio, F., Circella, M., De Marzo, C., De Pascale, M. P., Francke, T., Finetti, N., Golden, R. L., Grimani, C., Hof, M., Menn, W., Mitchell, J. W., Morselli, A., Ormes, J. F., Papini, P., Piccardi, S., Picozza, P., Ricci, M., Schiavon, P., Simon, M., Sparvoli, R., Spillantini, P., Stephens, S. A., Stochaj, S. J., Streitmatter, R. E., Suffert, M., Vacchi, A., Weber, N., and Zampa, N.: Measurements of Ground-Level Muons at Two Geomagnetic Locations, Phys. Rev. Lett., 83, 4241, doi:10.1103/PhysRevLett.83.4241, 1999.

Lesparre, N., Gibert, D., Marteau, J., D’eclais, Y., Carbone, D., and Galichet, E.: Geophysical muon imaging: feasibility and limits, Geophys. J. Int., 183, 1348, doi:10.1111/j.1365246X.2010.04790.x, 2010.

Lipari, P.: Lepton spectra in the earth's atmosphere, Astropart. Phys., 1, 195, doi:10.1016/0927-6505(93)90022-6, 1993.

Lipari, P. and Stanev, T.: Propagation of multi-TeV muons, Phys. Rev., D44, 3543, doi:10.1103/PhysRevD.44.3543, 1991.

Nakamura, K. et al. - Particle Data Group: Review of Particle Physics, J. Phys., G37, 075021, doi:10.1088/09543899/37/7A/075021, 2010.

Rastin, B. C.: An accurate measurement of the sea-level muon spectrum within the range 4 to $3000 \mathrm{GeV} / \mathrm{c}$, J. Phys. G., 10, 1609, doi:10.1088/0305-4616/10/11/017, 1984.

Selvi, M. - LVD Coll.: Annual muon flux modulation for $E_{\mu}>1.3 \mathrm{TeV}$ by LVD experiment, Proc. 31 st ICRC, paper: icrc0766, Lodz, Poland, 2009.

Sherman, N.: Atmospheric temperature effect for $\mu$ mesons observed at a depth of 846 m.w.e, Phys. Rev., 93, 1573, doi:10.1103/PhysRev.93.208, 1954.

Wilson, B. G.: A study of $\mu$-mesons incident at large zenith angles, Can. J. Phys., 37, 19, doi:10.1139/p59-004, 1959.

Zatsepin, G. T. et al. - MSU Coll.: Izv. Ross. Akad. Nauk, Sez. Fiz., 58, 119, 1994. 